\title{
Erratum to: Diffusion-weighted magnetic resonance imaging in the depiction of gastric cancer: initial experience
}

\author{
Lei Tang, ${ }^{1}$ Ying-Shi Sun, ${ }^{1} \mathrm{Zi}-Y u$ Li, ${ }^{2}$ Kun Cao, ${ }^{1}$ Xiao-Yan Zhang, ${ }^{1}$ Xiao-Ting Li, ${ }^{1}$ \\ Jia-Fu $\mathrm{Ji}^{2}$ \\ ${ }^{1}$ Department of Radiology, Key Laboratory of Carcinogenesis and Translational Research (Ministry of Education), Peking \\ University Cancer Hospital \& Institute, No.52 Fu Cheng Road, Hai Dian District, Beijing 100142, China \\ ${ }^{2}$ Department of Gastrointestinal Surgery, Key Laboratory of Carcinogenesis and Translational Research (Ministry of Education), \\ Peking University Cancer Hospital \& Institute, No.52 Fu Cheng Road, Hai Dian District, Beijing 100142, China
}

\section{Erratum to: Abdom Radiol (2016) 41:2-9 DOI: $10.1007 / \mathrm{s} 00261-015-0594-6$}

The original version of this article unfortunately contained a mistake. Ying-Shi Sun was not listed as one of the corresponding authors in the PDF version of this article. Lei Tang and Ying-Shi Sun are both corresponding authors for this article. Ying-Shi Sun is now included as a corresponding author with this erratum.

The online version of the original article can be found under doi: 10.1007/s00261-015-0594-6.

Correspondence to: Lei Tang; email: terrytang78@163.com; Ying-Shi Sun; email: sys27@163.com 